\section{Oral fluorescein angiography with the scanning laser ophthalmoscope in diabetic retinopathy: a case controlled comparison with intravenous fluorescein angiography}

D Squirrell', S Dinakaran', S Dhingra², C Mody', C Brand' and J Talbot'
${ }^{1}$ Department of Ophthalmology Royal Hallamshire Hospital Sheffield, UK

${ }^{2}$ Birmingham and Midland Eye Centre City Hospital Birmingham, UK

Correspondence: JF Talbot Department of Ophthalmology ,Royal Hallamshire Hospital Sheffield, UK.

Tel: + 44 (0) 1142713056 Fax: + 44 (0) 1142713682 E-mail: john.talbot@ sth.nhs.uk

Since its first description in 1961, ${ }^{1}$ ocular fundus photography with the intravenous administration of the sodium fluorescein has gained widespread acceptance as the gold standard investigation for visualization of the retinal capillary circulation. However, intravenously administered sodium fluorescein has a number of well-documented side effects ranging from mild nausea and itch (reported incidence $20 \%$ ) to the rare but more severe
Received: 30 November 2003

Accepted: 9 February 2004 Published online: 21 May 2004

The authors have no commercial or proprietary interests in any of the products mentioned. 
reactions of anaphylactic shock and acute myocardial infarction (reported incidence $<0.05 \%$ ). ${ }^{2}$ In patients technically or otherwise unsuitable for intravenous injections, oral administration of sodium fluorescein has been employed. ${ }^{3,4}$ Although the oral administration of sodium fluorescein has a lower incidence of side effects than intravenous administration, ${ }^{5}$ the contrast of the subsequent angiogram is often disappointing and this limits the usefulness of the investigation.

The confocal scanning laser ophthalmoscope (SLO) is an imaging system that can produce high-resolution images of the retina and its circulation. An image capture system that utilizes the SLO, rather than a conventional fundus camera system, may overcome many of the technical difficulties that have previously been encountered with oral fluorescein angiography. It has been demonstrated that this technique can produce images that are of sufficient quality to diagnose and treat many types of retinal pathology. ${ }^{6}$ The aim of this study was to investigate whether oral fluorescein angiography with the SLO can produce angiograms that are of sufficient quality to investigate patients with sightthreatening diabetic retinopathy. We report the findings of a prospective study comparing oral fluorescein angiography using the SLO with intravenous fluorescein angiography using the conventional fundus camera, in patients with sight-threatening diabetic retinopathy.

\section{Patients and methods}

Ethical approval was granted prior to the commencement of this study. All patients were recruited from the hospital diabetic eye clinic, and consecutive patients whose diabetic retinopathy required intravenous fundus fluorescein angiography (IVFFA) were approached. Consenting patients were examined by one of the investigators not involved in reading the subsequent angiograms and the level of the patient's retinopathy and maculopathy in both eyes was recorded. The levels of diabetic retinopathy and clinically significant macular oedema (CSMO) were defined according to ETDRS criteria. ${ }^{7,8}$ Patients underwent IVFFA on the day of the clinic, returned a week later when the oral fluorescein angiogram was performed. Exclusion criteria were pregnancy, a personal history of asthma, and a personal or family history of allergy to any contrast dye.

\section{Angiography protocols}

Both the intravenous and oral fluorescein angiograms were conducted with dilated pupils. Pupillary dilatation was achieved with tropicamide $1 \%$ and phenylepherine $2.5 \%$ eye drops in all cases.
IVFFA

The intravenous angiogram was conducted using a Zeiss FF5 $30^{\circ}$ fundus camera loaded with $35 \mathrm{~mm}$ negative film (Ilford HP5 black and white film). Following the bolus

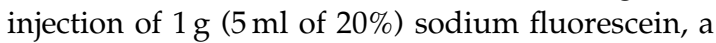
standard rapid stereo sequence was performed of both maculae until the arteriovenous phase reached.

Thereafter, a modified ETDRS survey of the peripheral retina was performed. Further, late-phase photographs were taken 2 and 5 min later to complete the sequence.

\section{Oral fluorescein angiography}

The oral fluorescein angiogram was performed using the Heidleberg retina angiograph with a confocal SLO. The sodium fluorescein was administered orally using gel capsules taken with water, each containing $250 \mathrm{mg}$ sodium fluorescein (Manderville Medicines, UK). The dose of sodium fluorescein required to produce the optimal oral angiogram has been shown to be $25-30 \mathrm{mg} / \mathrm{kg}$ body weight. ${ }^{9}$ Based on this recommendation, patients received a dose of $25 \mathrm{mg} / \mathrm{kg}$ rounded up to the nearest $250 \mathrm{mg}$ with a maximum dose of $2.5 \mathrm{~g}$. Following ingestion of the capsules, a sequence of electronic images was taken with the SLO. The image sequence was identical to that followed for the IVFFA. Images were recorded at time points $0,5,10,15,20,25,30,35,40,45,50$, and 55 min after ingestion, and the images formed were stored on the hard drive. The time at which the dye was first visualized in the retinal circulation and the time for the optimal oral angiogram to be obtained were noted in each case.

\section{Angiogram analysis}

The angiograms were reported by an experienced medical retina specialist (angiogram reader 1) using a standard proforma. In the first instance, all the oral angiograms were reported and after a period of 1 month the process was repeated for the intravenous angiograms. Angiogram reader 1 was asked to grade six facets of each angiogram: visualization of the foveal avascular zone (FAZ); the resolution of the angiogram; the absence or presence of macular leakage; the absence or presence of microaneurysms within an area of macular leakage; the absence or presence of neovascularization of the disc (NVD) or elsewhere (NVE); the absence or presence of significant confluent nonperfusion (greater than 10 disc diameters of confluent nonperfusion). The proforma was also used by a second, independent, medical retina specialist (angiogram reader 2) who was asked to report on the visualization of the FAZ in the set of intravenous fluorescein angiograms. Angiogram reader 2 was masked from the results of the previous reader's interpretation of the FAZ. 
The system used to analyse and compare all angiograms was based on a modified version of that previously used by Garcia et al. ${ }^{6}$ Visualization of the FAZ was graded on a three-point grading system: grade 1, FAZ seen and intact; grade 2, FAZ seen but not intact; grade 3, not possible to judge FAZ on this angiogram. The resolution of the angiogram was graded according to the smallest order of branch retinal artery seen in the temporal arcades. A five-point grading system was used: grade 0, first-order branch not visualized; grade 1; firstorder branch only clearly visualized, grade 2 , secondorder branch clearly visualized; grade 3, third-order branch clearly visualized; grade 4 , fourth-order branch clearly visualized. Macular leakage, macular microaneurysms, neovascular disease, and significant confluent nonperfusion were all graded using a threepoint grading system: grade 1, not seen; grade 2; seen, grade 3 , cannot judge from this angiogram.

\section{Patient preference survey}

After completing the study, patients were asked to complete a short questionnaire with the aim of establishing if they had any preference between the two angiography techniques. As it was recognized that the experimental oral angiography protocol was very intensive, patients were asked to complete the questionnaire twice. On the first time of answering, they were asked to give their response based on the experimental oral angiography protocol they had just experienced. Patients were then asked for their response supposing an abridged oral angiography protocol with a reduced number of scanning episodes had been used.

\section{Statistical analysis}

The levels of agreement (Kappa) between the first angiogram readers' reports of the intravenous and oral angiograms were determined. A Kappa statistic was also calculated to examine the level of agreement between the two reports generated by the independent specialists when asked to judge the visualization of the FAZ on the same set of intravenous angiograms. The intra and interobservational variation between the i.v. and oral angiograms were then compared. For the purpose of this study, a Kappa statistic of 0.80 or more was taken as an indicator of excellent clinical agreement, values between 0.61 and 0.80 were regarded as substantial clinical agreement, and values between 0.41 and 0.60 were regarded as indicating moderate agreement. ${ }^{10}$

\section{Results}

A total of 25 patients were enrolled into the study. As a consequence of advanced diabetic retinopathy, one patient had a blind right eye. The study therefore comprised 49 angiograms. No patients withdrew from the study once enrolled. The category of maculopathy and retinopathy recorded in each of the 49 eyes is summarized in Table 1. No patient experienced any reaction to intravenous angiography. Four patients experienced mild indigestion after taking the oral fluorescein capsules. Of these, three had ingested all the capsules in the same mouthful. Patients were subsequently advised to take the capsules individually. Thereafter, only one patient of 16 experienced indigestion after taking the capsules. Aside from indigestion, no other side effects were experienced after oral angiography.

\section{Angiogram analysis: resolution of the angiograms}

The median time for dye entry into the retinal circulation after ingestion of the capsules was 20 min (range 10$45 \mathrm{~min}$ ). The median time to optimal angiogram after ingestion of the capsules was $35 \mathrm{~min}$ (range 25-55 min). The results of the smallest order branch retinal arteriole seen in both sets of angiograms are summarized in Table 2. Angiogram resolution, as judged by smallest order branch arteriole seen, was graded grade 4 in 42 oral $(86 \%)$ and $42(86 \%)$ intravenous angiograms. No

Table 1 Summary of the clinical diagnosis of patients enrolled in the study

\begin{tabular}{lc}
\hline $\begin{array}{l}\text { Diagnosis (maculopathy and retinopathy status } \\
\text { of each eye) }\end{array}$ & $\begin{array}{c}\text { Number of } \\
\text { eyes }(\mathrm{N}=49)\end{array}$ \\
\hline No CSMO; mild/moderate NPDR & 2 \\
No CSMO; severe/very severe NPDR & 4 \\
CSMO; mild/moderate NPDR & 8 \\
CSMO; severe/very severe NPDR & 23 \\
CSMO; previously treated PDR & 2 \\
CSMO; proliferative DR & 10 \\
\hline
\end{tabular}

NPDR: nonproliferative diabetic retinopathy (from ETDRS ${ }^{7}$ ); CSMO: clinically significant macular oedema (from ETDRS ${ }^{8}$ );

Table 2 Summary of the angiograms resolution as judged by smallest order branch retinal arteriole seen in the temporal arcades

\begin{tabular}{lccccc}
\hline & \multicolumn{5}{c}{ Oral angiograms } \\
\cline { 2 - 6 } & Grade 4 & Grade 3 & Grade 2 & Grade 1 or 0 & Total \\
\hline i.v. angiograms & & & & & \\
Grade 4 & 40 & 0 & 2 & 0 & 42 \\
Grade 3 & 2 & 2 & 0 & 0 & 4 \\
Grade 2 & 0 & 3 & 0 & 0 & 3 \\
Grade 1 or 0 & 0 & 0 & 0 & 0 & 0 \\
Total & 42 & 5 & 2 & 0 & 49 \\
\hline
\end{tabular}


angiogram in either set was judged to be less than grade 2 quality.

Angiogram analysis: visualization of the foveal avascular zone

Angiogram reader 1 judged that the FAZ was seen and intact in seven intravenous angiograms, seen and disrupted in 24 intravenous angiograms, and was not seen in 18 intravenous angiograms. Angiogram reader 2 judged that the FAZ was seen and intact in three intravenous angiograms, seen and disrupted in 27 angiograms, and was not seen in 19 intravenous angiograms. The Kappa statistic for the level of agreement between the two observers judging the FAZ on the intravenous angiograms was 0.73 (95\% CI 0.55$0.90)$ (Table 3). Angiogram reader 1 was also asked to report the oral angiograms. With oral angiography the FAZ was seen and judged intact in five angiograms, seen and judged to be disrupted in five angiograms (Figure 1), and was not seen in 39 angiograms. The Kappa statistic for the level of agreement between the same observer judging the FAZ in the intravenous and oral angiograms was 0.10 (95\% CI 0-0.30) (Table 3).

\section{Angiogram analysis: macular leakage and identification of macular microaneurysms within areas of leakage}

Macular leakage from microaneurysms was identified in 43 intravenous angiograms and 45 oral angiograms (Figure 2). The Kappa statistic for the level of agreement between the same observer reading the intravenous and oral angiograms for both macular leakage and the identification of macular microaneurysms within an area of macular leakage was 0.78 (95\% CI 0.72-0.83) (Table 3). A review of the data recorded at clinical examination revealed that oral angiography correctly identified clinically significant macular oedema (CSMO) in all 43 eyes in which it was present. Oral angiography revealed macular leakage from perifoveal microaneurysms in two eyes that did not have CSMO.

\section{Angiogram analysis: significant peripheral nonperfusion}

Of the 43 eyes that had a full survey of the peripheral retina with intravenous angiography, an area of confluent peripheral nonperfusion measuring 10 disc diameters or more was identified in 29. A total of 10 intravenous angiograms revealed confluent peripheral nonperfusion measuring less than 10 disc diameters. Four intravenous angiograms were not of sufficient quality to make a reliable judgment. Examination of the oral angiograms of these corresponding 43 eyes revealed that confluent nonperfusion measuring 10 disc diameters or more was

Table 3 The level of agreement between different facets of the angiogram

\begin{tabular}{lc}
\hline Facet of angiogram analysed & Kappa \\
statistic \\
$(95 \% \mathrm{CI})$
\end{tabular}

Visualization of the FAZ: intravenous angiogram reader 1 versus intravenous angiogram reader 2

0.73

Visualization of the FAZ: intravenous angiogram reader 1 versus oral angiogram reader 1

$(0.55-0.90)$

Macular oedema: intravenous angiogram reader 1 versus oral angiogram reader 1

0.78

Identification of microaneurysms in areas of macular leakage: intravenous angiogram reader 1 versus oral angiogram reader 1

$(0.72-0.82)$

Significant nonperfusion: intravenous angiogram reader 1 versus oral angiogram reader 1

Proliferative diabetic retinopathy: intravenous 1.0 angiogram reader 1 versus oral angiogram reader 1


Figure 1 i.v. FFA (left) and oral FFA (right) of the same patient demonstrating visualization of a disrupted FAZ. 
observed in 23 angiograms (Figure 3). In all, 12 oral angiograms revealed confluent peripheral nonperfusion measuring less than 10 disc diameters and eight oral angiograms were not of sufficient quality to make a reliable judgment. The Kappa statistic for the level of agreement between the same observer reading the intravenous and oral angiograms for significant peripheral nonperfusion was 0.54 (95\% CI 0.42-0.66) (Table 3). Of the eight oral angiograms that were not of sufficient quality to make a reliable judgment about peripheral nonperfusion, five had profuse dye leakage from neovascular complexes, which obscured the view of the peripheral retinal circulation (Figure 2). If the five angiograms in which this occurred were excluded, then oral angiography identified 23 of the 24 eyes in which significant nonperfusion was found on IVFFA.

\section{Angiogram analysis: proliferative diabetic retinopathy}

Proliferative diabetic retinopathy was identified in 11 eyes by intravenous angiography. The presence of proliferative diabetic retinopathy (isolated NVE) had been missed clinically in one of these patients. There was complete agreement between the intravenous and oral angiograms regarding the presence or absence of proliferative diabetic retinopathy.

\section{Patient preference survey}

Using the study oral angiography protocol, 16/25 (64\%) patients much preferred the oral to the intravenous angiogram, with the remainder expressing no preference. No patient expressed a preference for intravenous angiography. When asked which of the two angiography techniques they would rather undergo if an abridged oral angiogram protocol had been used that required a reduced number of scanning episodes, the number of patients who much preferred the oral technique was 23/ $25(92 \%)$. The remaining two patients expressed no preference.

\section{Discussion}

Oral angiography with SLO was first described by Garcia et $a l .^{6}$ In a study comparing the technique with
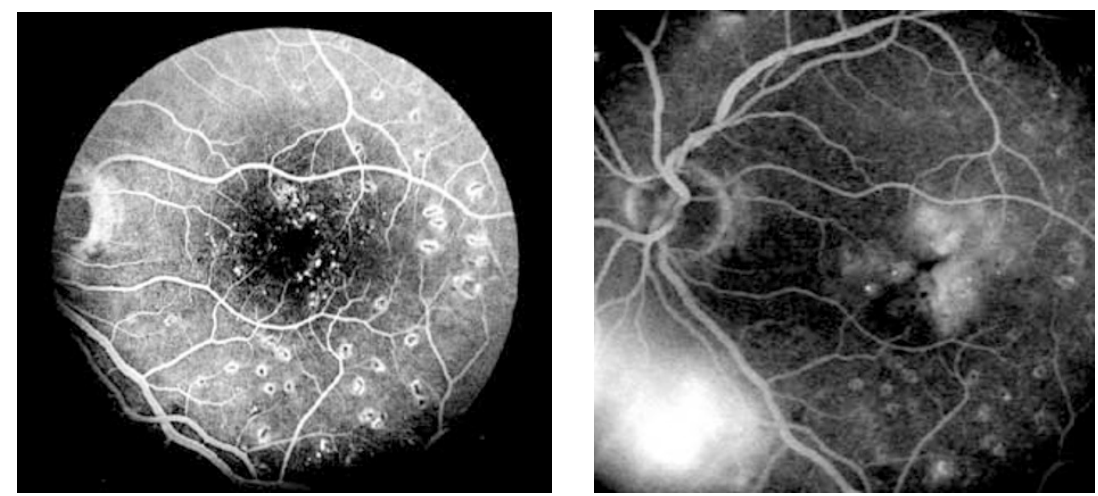

Figure 2 Illustration of the difficulties that can be encountered with oral FFA when assessing the FAZ in a patient with mixed maculopathy (right-hand picture). The corresponding IVFFA is shown for comparison. (Note the masking effect in the periphery from the NVE's and the visualization of the leaking microaneurysms within the area of macular leakage on the oral angiogram.)
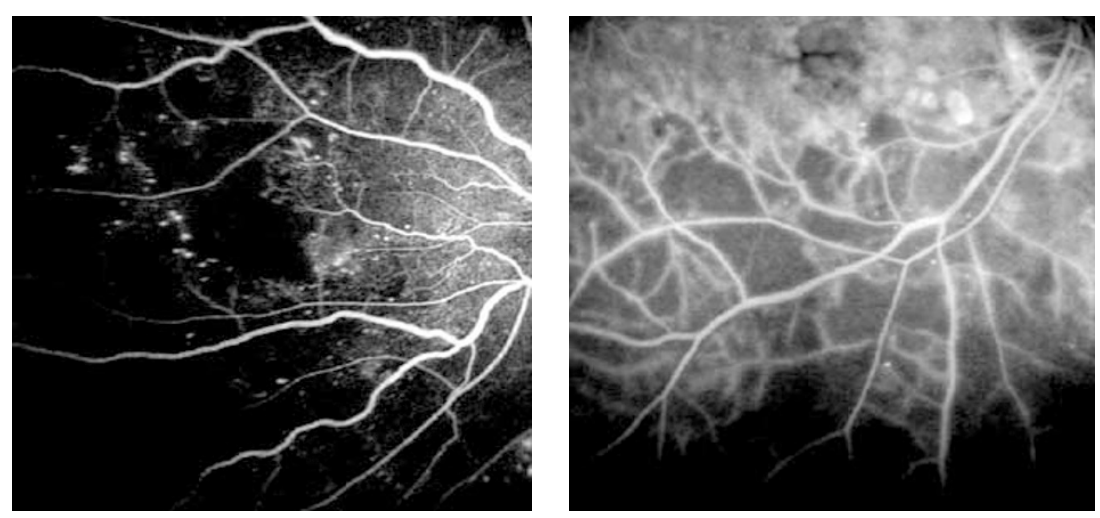

Figure 3 Oral FFAs illustrating peripheral nonperfusion in two different patients. 
conventional IVFFA, they concluded that the image obtained by oral angiography was of sufficient quality to diagnose and treat a variety of retinal pathologies. Although promising, the range of heterogeneous retinovascular disease examined in this study meant that the efficacy of the technique in investigating individual diseases remained unproven. The purpose of the current study was to evaluate how the technique of oral angiography with the SLO compares with the current 'gold standard', IVFFA with a fundus camera, in the investigation of sight-threatening diabetic retinopathy.

We, like Garcia et $a l^{6}{ }^{6}$ found that the resolution of oral angiography with the SLO was, in most cases, equal to that of conventional intravenous angiography. The retinal circulation was best visualized after $30-40 \mathrm{~min}$ in most patients and this time delay was similar to that previously reported by the other group that has used gel capsules for the oral administration of fluorescein. ${ }^{9}$ This compares to a time delay of just $15-30$ min reported in the study conducted by Garcia $e t a l^{6}$ who used a liquid preparation of fluorescein. While the time to dye entry may be longer with gel capsules, our results suggest that the subsequent oral angiogram is of equal quality regardless of how the dye is administered.

Judgments about the integrity of the FAZ with angiography can be highly subjective. Therefore, to be confident that the report of the FAZ from the intravenous angiograms was a reliable benchmark against which the oral angiograms could be judged, two independent readers were asked to perform this assessment. As there was substantial clinical agreement between these two angiogram readers (Kappa 0.73), we concluded that angiogram reader one's assessment of the FAZ on intravenous angiography was reliable and accurate. Compared to intravenous angiography, oral angiography proved to be an unreliable method for visualizing the FAZ. The principle cause for the technique's poor performance was the high number of cases where macular leakage masked the underlying capillary details of the central macula (Figure 2). Our findings are contrary to those reported by Garcia et al, ${ }^{6}$ who reported that although the percentage of the FAZ seen with oral angiography was less than that seen with intravenous angiography, the difference was not significant. This discrepancy can probably be explained by differences in the cohorts studied as most eyes examined in our study had macular oedema, whereas most eyes in the previous study did not. As oral fluorescein angiography can only provide late venous phase images, any macular leakage will impair visualization of the FAZ. We therefore conclude that, in the context of diabetic retinopathy, oral fluorescein angiography is not a reliable tool for investigating foveal ischaemia particularly when there is coexisting macular oedema.
In contrast, macular leakage, the identification of microaneurysms within an area of macular leakage, and leakage from neovascular complexes were all extremely well seen with oral angiography, and there was substantial clinical agreement between the oral and intravenous angiogram reports in these three respects. These findings support those previously published by Garcia et $a l^{6}$, who also found that dye leakage was well visualized with oral fluorescein angiography. Oral fluorescein angiography therefore appears to be a useful tool for detecting and assessing macular oedema and proliferative diabetic retinopathy.

Profuse dye leakage from neovascular complexes impaired visualization of the peripheral retina and this complicated the analysis of peripheral nonperfusion. The difficulty in visualizing the peripheral retina in those eyes with proliferative diabetic retinopathy is a product of two characteristics of the image that is obtained with oral angiography: firstly, the image is a late venous phase image and thus dye leakage is prominent; secondly, the image is typically of lower contrast than that seen with IVFFA and consequently the images from the periphery are more susceptible to being degraded by dye leakage from elsewhere (Figure 2). Visualization of peripheral nonperfusion in cases where dye leakage from neovascular complexes did not obscure the periphery was otherwise comparable with IVFFA. (In this scenario, 23 out of 24 eyes with significant nonperfusion on IVFFA were found to have significant nonperfusion on oral angiography.) We conclude that oral angiography with the SLO is not a reliable tool for detecting peripheral nonperfusion in the presence of proliferative diabetic retinopathy. It may however be a useful tool for detecting peripheral nonperfusion in eyes with nonproliferative diabetic retinopathy.

There are two potential weaknesses in this study. Firstly, it was not possible to mask the angiogram reader from the type of angiogram being reported. Secondly, the results from just one angiogram reader were used to compare the set of oral and intravenous angiograms. These weaknesses raise the possibility of observer bias. In order to minimize this, the angiogram reader was requested to read the oral angiograms first and in isolation of the clinical findings. As an additional safeguard, the intravenous angiograms were withheld from the angiogram reader for a month and when they were presented it was in a different sequence. Although the potential for observer bias remains, we are confident that these precautions mean that our results are an accurate representation of the performance of these two angiographic techniques.

This small study has demonstrated that oral angiography with the SLO may be a useful tool for investigating selected patients with sight-threatening 
diabetic retinopathy, but what role could the technique play in the management of such patients? We found that oral angiography can identify leaking microaneurysms in patients with CSMO. The technique also identified all cases where NVD or NVE was present. However, oral angiography with the SLO proved to be unreliable in detecting foveal ischaemia, and the value of the technique in identifying peripheral nonperfusion is controversial. These results suggest that oral angiography with the SLO is unlikely to replace conventional intravenous angiography in the routine investigation of diabetic eye disease. However, oral angiography with the SLO did detect all patients with treatable sight-threatening diabetic retinopathy (CSMO, NVE, NVD). It also stratified patients into different risk groups based upon the presence or otherwise, of peripheral nonperfusion and NVE/NVD. One possible application of oral angiography with the SLO may therefore lie in the screening of patients with high-risk diabetic retinopathy, and identifying those patients who need further assessment and treatment and those who do not. Increasingly, techniques that will objectively assess sight-threatening diabetic retinopathy as part of a structured screening programme are being sought. ${ }^{11,12}$ Oral angiography is convenient and acceptable to patients and therefore meets many of the criteria that such a system must fulfill.

\section{Conclusion}

Our findings demonstrate that oral angiography with the SLO can provide the clinician with consistently highquality angiograms that allow judgments to be made about the presence of treatable diabetic maculopathy, proliferative diabetic retinopathy and, in the absence of NVE and NVD, peripheral nonperfusion. The principal limitation of the technique is that it can only provide latephase pictures and as such it cannot be relied upon to demonstrate foveal ischaemia, particularly in the presence of coexisting macular oedema. Oral angiography is therefore unlikely to replace intravenous angiography in the investigation of the majority of patients with diabetic eye disease. The technique may offer the opportunity to assess objectively patients with potentially sight-threatening diabetic retinopathy as part of a structured screening programme.

\section{References}

1 Novotny HR, Alvis DL. A method of photographing fluorescence in circulating blood in the human retina. Circulation 1961; 24: 82-86.

2 Yannuzzi LA, Rohrer KT, Tindel LJ, Sobel RS, Costanza MA, Shields W, Zang E. Fluorescein angiography complication survey. Ophthalmology 1986; 93: 611-617.

3 Nayak B, Ghose S. A method for fundus evaluation in children with oral fluorescein. Br J Ophthalmol 1987; 71: 907-909.

4 Noble M, Cheng H, Jacobs P. Oral fluorescein and cystoid macular edema: detection in aphakic and pseudophakic eyes. Br J Ophthalmol 1984; 68: 221-224.

5 Hara T, Inami M, Hara T. Efficacy and safety of fluorescein angiography with orally administered sodium fluorescein. Am J Ophthamol 1998; 126: 560-564.

6 Garcia CR, Rivero ME, Bartsch D, Ishiko S, Takamiya A, Fukai $\mathrm{H}$ et al. Oral fluorescein angiography with the confocal scanning laser ophthalmoscope. Ophthalmology 1999; 106: 1114-1118.

7 ETDRS Report No 10. Grading diabetic retinopathy from stereoscopic color fundus photographs - an extension of the modified Airlie house classification. Ophthalmology 1991; 98: 786-806.

8 Early treatment diabetic retinopathy study group. Photocoagulation for diabetic macular edema. Arch Ophthalmol 1985; 103: 1796-1806.

9 Watson AP, Rosen ES. Oral Fluorescein angiography: reassessment of its relative safety and evaluation of optimum conditions with use of capsules. Br J Ophthalmol 1990; 74: 458-461.

10 Dixon R, Munro J, Silcocks P. The evidence Based Medicine Workbook. Butterworth Heinmann: Oxford, 1997, pp 11-12, (ISBN 075062590 2).

11 Newsome R, Moate B, Casswell T. Screening for diabetic retinopathy using digital colour photography and oral fluorescein angiography. Eye 2000; 14: 579-582.

12 Newsome R, Sinthanayothin C, Boyce J, Casswell G, Williamson TH. Clinical evaluation of 'local enhancement' for oral fluorescein angiograms. Eye 2000; 14: 318-323. 